\title{
Evolutionary Algorithm for Identifying Discontinuous Parameters of Inverse Problems
}

\author{
Zhijian Wu, Dazhi Jiang, and Lishan Kang \\ The State Key Laboratory of Software Engineering, \\ Wuhan University, Wuhan, China \\ \{zjwu9551, jiangdazhi111007\} @sina.com
}

\begin{abstract}
In this paper, we make an investigation into the discontinuous parameter identification in the case of elliptic problem. The discontinuous parameter is identified by evolutionary algorithm for the first time. For this kind of problem, we present a two-level evolutionary algorithm. The first level is the evolution for discontinuous point and the second level is the evolution for parameter. The numerical experiments suggest that the algorithm carries such features as good stability and adaptability and is not very sensitive to the noise in observation data as well.
\end{abstract}

Keywords: Parameter Identification, Inverse Problem, Evolutionary algorithms.

\section{Introduction}

The major purpose of this paper aims to propose an evolutionary algorithm in the identification of the unknown coefficient $q(x)$ in the case of elliptic problem. We will take into consideration the case when the coefficient $q(x)$ is discontinuous.

$$
\left\{\begin{array}{l}
-\frac{d}{d x}\left(q(x) \frac{d}{d x} u(x)\right)=f(x) \text { in } \Omega \\
u(x)=0 \text { on } \Gamma
\end{array}\right.
$$

Here the $\Omega$ can be any bounded domain in $R$, with piece wise smooth boundary $\Gamma$ and $f(x)$ being given.

So far, a lot of algorithms for solving the continuous parameter identification problem have been worked out, among which, three kinds of methods can be used. One of them is termed as the traditional mathematic and physical methods [1-5]. The second one is known as the evolutionary algorithms [6-10], and the numerical experiments indicate that these algorithms are good to solve inverse problem. The third one is to model parameter function by Genetic Programming [11-14]. But much less work has been done on the identification of discontinuous parameters [15]. So in this paper, we investigate the discontinuous parameter identification and the discontinuous parameter is identified by evolutionary algorithm for the first time. The paper is presented as follows. In the second section, the new algorithm is described. Some numerical experiments are described in the third section and in the fourth section some conclusions have been drawn. 


\section{Description of Algorithm}

As an inverse problem in elliptic systems, parameter identifying is the process to find the potential solution $q^{*}(x)$ that makes $u_{q^{*}(x)}$ match the observation data of $u(x)$ as optimally as possible, where we obtain $u_{q^{*}(x)}$ by solving equation (1) with $q^{*}(x)$.

To put it simpler, the following elliptic problem can be considered.

$$
\left\{\begin{array}{l}
-\frac{d}{d x}\left(q(x) \frac{d}{d x} u(x)\right)=f(x), x \in(0,1) \\
u(0)=u(1)=0
\end{array}\right.
$$

We consider the case in which the $q(x)$ has one discontinuous point, which can be expanded to multi discontinuous point case. As for this kind of problem, we present a two-level evolutionary algorithm. The first level is the evolution for discontinuous point and the second level is the evolution for $q(x)$.

The interval $[0,1]$ is divided equally into $n$ parts, while the step size $h=1 / n$, and mesh point $x_{i}=i h(i=0,1, \ldots, n)$. Suppose we have the observation data of $u(x)$ at mesh point $x_{i}(i=1,2, \ldots, n-1)$, two boundary points values are given. Here the observation data of $u(x)$ can be denoted as $\tilde{u}_{o b}=\left(\tilde{u}_{1}, \tilde{u}_{2}, \ldots, \tilde{u}_{n-1}\right)$.

In the first level evolution, we denote the individual as $d_{i}\left(i=1,2, \ldots, N_{d}\right)$. For individual $d_{i}$ (for the sake of convenience, we suppose the $d_{i}$ is just the same as the some node $x_{l}$ ), we conduct the second level evolution (i.e. the evolution of $q(x)$ ). Suppose $d_{i}=x_{l}=l^{*} h$, we construct the following base functions:

$$
\begin{aligned}
& \varphi_{0}(x)=\left\{\begin{array}{ll}
\frac{x_{1}-x}{h}, & x \in\left[x_{0}, x_{1}\right], \\
0, & \text { otherwise. }
\end{array} \quad \varphi_{i}(x)= \begin{cases}\frac{x-x_{i-1}}{h}, & x \in\left[x_{i-1}, x_{i}\right], \\
\frac{x_{i+1}-x}{h}, & x \in\left[x_{i}, x_{i+1}\right], i=1,2, \ldots, l-1 \\
\text { otherwise. }\end{cases} \right. \\
& \varphi_{l}^{(1)}(x)=\left\{\begin{array}{ll}
\frac{x-x_{l-1}}{h}, & x \in\left[x_{l-1}, x_{l}\right], \\
0, & \text { otherwise. }
\end{array} \varphi_{l}^{(2)}(x)= \begin{cases}\frac{x_{l+1}-x}{h}, & x \in\left[x_{l}, x_{l+1}\right], \\
0, & \text { otherwise. } .\end{cases} \right. \\
& \varphi_{i}(x)= \begin{cases}\frac{x-x_{i-1}}{h}, & x \in\left[x_{i-1}, x_{i}\right], \\
\frac{x_{i+1}-x}{h}, & x \in\left[x_{i}, x_{i+1}\right], i=l+l+2, \ldots, 1 \\
0, & \text { otherwise. }\end{cases} \\
& \varphi_{n}(x)= \begin{cases}\frac{x-x_{n-1}}{h}, & x \in\left[x_{n-1}, x_{n}\right], \\
0, & \text { otherwise. }\end{cases}
\end{aligned}
$$

$\operatorname{Set} q(x)=\sum_{i=0}^{l-1} q_{i} \varphi_{i}(x)+q_{l}{ }^{(1)} \varphi_{l}{ }^{(1)}(x)+q_{l}{ }^{(2)} \varphi_{l}{ }^{(2)}(x)+\sum_{i=l+1}^{n} q_{i} \varphi_{i}(x)$ 
where $q_{l}^{(1)}=\lim _{x \rightarrow x_{l}^{-}} q(x), q_{l}^{(2)}=\lim _{x \rightarrow x_{l}^{+}} q(x)$.

Identification of $q(x)$ is converted to that of the discrete values $q=\left(q_{0}, q_{1}, \ldots, q_{l}^{(1)}, q_{l}^{(2)}, \ldots, q_{n}\right)$. So for the second level evolution, we denote the individual as $\left(q_{0}, q_{1}, \ldots, q_{l}{ }^{(1)}, q_{l}{ }^{(2)}, \ldots, q_{n}\right)$. For each individual $d_{i}$ we evolve it to get the best $q_{d_{i}}(x)$. For this $q_{d_{i}}(x)$, we can get $u_{d_{i}}(x)$ by solving equation (2). The fitness of individual $d_{i}$ is defined as:

fitness $\left(d_{i}\right)=h \sum_{i=1}^{n-1}\left(\bar{u}_{d_{i}}\left(x_{i}\right)-\widetilde{u}_{i}\right)^{2}+\beta\left(\sum_{i=1}^{l-1}\left|q_{i}-q_{i-1}\right|+\left|q_{l}{ }^{(1)}-q_{l-1}\right|+\left|q_{l+1}-q_{l}{ }_{l}^{(2)}\right|+\sum_{i=1+2}^{n}\left|q_{i}-q_{i-1}\right|\right)$

For two individuals $d_{1}$ and $d_{2}$, if fitness $\left(d_{1}\right)<$ fitness $\left(d_{2}\right)$ it is said that $d_{1}$ is better than $d_{2}$.

The following operators will be adopted in our algorithm.

\section{Smooth operator}

\section{Begin}

For $i=1$ to $l-1$ do $q_{i}=\left(q_{i-1}+q_{i}+q_{i+1}\right) / 3$;

For $i=l+1$ to $n-1$ do $q_{i}=\left(q_{i-1}+q_{i}+q_{i+1}\right) / 3$;

\section{End}

\section{Multiple parent crossover operator}

The multiple parent crossover operator has been used in our algorithm, it plays an essential role in keeping the diversity of population, and making the parents' merits inherited by their offspring as well. The crossover operator is described as follows (suppose there are $N$ individuals in population $P$ ).

\section{Procedure multiple parents crossover operator}

\section{Begin}

Step 1: Select M individuals $\vec{q}^{(1)}, \vec{q}^{(2)}, \ldots, \vec{q}^{(M)}$ randomly from population $P$ to form a sub-space $V=\left\{\vec{q} \mid \vec{q} \in D^{n+1}, \vec{q}=\sum_{i=1}^{M} a_{i} \vec{q}^{(i)}\right\}$, where $a_{i}$ satisfies the condition $\sum_{i=1}^{M} a_{i}=1, a_{i} \in[-0.5,1.5]$;

Step 2: Produce a new individual $\vec{q}_{\text {new }}$ randomly in sub-space $V$.

End.

The algorithm for solving the discontinuous parameter problem is described as follows:

The first level evolutionary algorithm:

\section{Begin}

Step 1: $N_{d}$ individuals $d_{1}, d_{2}, \ldots, d_{N_{d}}$ are randomly and uniformly produced in the search space as to form the initial population $D^{(0)}$ and set $t_{d}=0$;

Step 2: For each $d_{i}$ conduct the second level evolutionary algorithm to get an individual $q_{d_{i}}$ corresponding to $d_{i}$.

Step 3: When terminative condition is satisfied with go to Step 5; 
Step 4: Genetic operations for $D^{\left(t_{d}\right)}$. Conduct multiple parent crossover operating, and produce a new individual $d_{\text {new }}$. Conduct the second level evolutionary algorithm for $d_{\text {new }}$. If the $d_{\text {new }}$ is better (i.e. the fitness is smaller) than the worst one in $D^{\left(t_{d}\right)}$ then substitute it for the worst one and form a new population $D^{\left(t_{d}+1\right)}$ else $D^{\left(t_{d}+1\right)}=D^{\left(t_{d}\right)} \cdot t_{d}=$ $t_{d}+1$. go to Step 3;

Step 5: Output the best individual $\mathrm{d}$ and the corresponding $q_{d}$.

\section{End}

The second level evolutionary algorithm

\section{Begin}

Step 1: $N_{q}$ individuals are randomly and uniformly produced in the search space as to form the initial population $P^{(0)}$ and set $t_{q}=0$;

Step 2: When terminative condition is satisfied with go to end;

Step 3: Genetic operations (crossover and smooth operation). Conduct multiple parent crossover operation, and produce a new individual. Conduct the smooth operator for the new individual. If the new individual is better (i.e. the fitness is smaller) than the worst one in $P^{\left(t_{q}\right)}$ then substitute it for the worst one and form a new population $P^{\left(t_{q}+1\right)}$ else $P^{\left(t_{q}+1\right)}=P^{\left(t_{q}\right)} \cdot t_{q}=t_{q}+1$. go to Step 2;

Step 4: Output the best solution.

\section{End}

In the following experiments, in the case of the first level evolution (discontinuous point evolution), the Popsize $N_{d}$ is 10 , the dimension $M$ of crossover subspace is 3 . As for the second level evolution $\left(q(x)\right.$ evolution), the Popsize $N_{q}$ is 100 , the dimension $M$ of crossover subspace is 10 .

As for the second level evolution, the terminate condition is

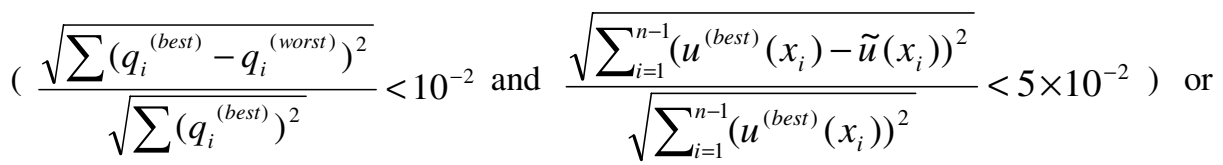

(the generation for the second level evolution $\geqq 20000$ ).

As for the first level evolution, the terminate condition is

$$
\left(\frac{\sqrt{\sum_{i=1}^{n-1}\left(u^{(b e s t)}\left(x_{i}\right)-\tilde{u}\left(x_{i}\right)\right)^{2}}}{\sqrt{\sum_{i=1}^{n-1}\left(u^{(b e s t)}\left(x_{i}\right)\right)^{2}}}<10^{-3}\right) \text { or (the generation for the first level }
$$

evolution $\geqq 8$ ).

\section{Numerical Experiments}

Example 1:

$$
\left\{\begin{array}{l}
\frac{d}{d x}\left(q(x) \frac{d}{d x} u(x)\right)=f(x), x \in(0,1) \\
u(0)=0, u(1)=0
\end{array}\right.
$$


Where $q(x)=\left\{\begin{array}{l}\pi, 0 \leq x \leq 0.5 \\ 1,0.5<x \leq 1\end{array}, u(x)=\left\{\begin{array}{l}x-\frac{1}{2}+\sin (\pi x), 0 \leq x \leq 0.5 \\ 1-\cos (\pi x), 0.5<x \leq 1\end{array}\right.\right.$

$$
\tilde{u}_{i}=\left(1+\delta_{i}\right) u\left(x_{i}\right) \text {, where } \delta_{i}(i=0,1, \ldots n-1) \text { is a random number in }(-\delta, \delta)
$$

Test 1-1: $\delta=0, \beta=10^{-4}$. Run time is 90 seconds. The times for evaluating the discontinuous point are 14. The times for evaluating $q$ are 522151. The discontinuous point is $0.500000, \frac{\sqrt{\sum_{i=1}^{n-1}\left(u^{(\text {best })}\left(x_{i}\right)-\widetilde{u}\left(x_{i}\right)\right)^{2}}}{\sqrt{\sum_{i=1}^{n-1}\left(u^{(b e s t)}\left(x_{i}\right)\right)^{2}}}=0.000070$.

In the following Figure, the line — presents the original function $q(x)$, while the line $\ldots$ presents the $q^{*}(x)$ identified by our algorithm.

Test 1-2: $\delta=1 \%, \beta=10^{-4}$. Run time is 190 seconds. The times for evaluating the discontinuous point are 26. The times for evaluating $q$ are 1042700. The noncontinuous point is $0.500000, \frac{\sqrt{\sum_{i=1}^{n-1}\left(u^{(\text {best }}\left(x_{i}\right)-\widetilde{u}\left(x_{i}\right)\right)^{2}}}{\sqrt{\sum_{i=1}^{n-1}\left(u^{(\text {best })}\left(x_{i}\right)\right)^{2}}}=0.006393$.

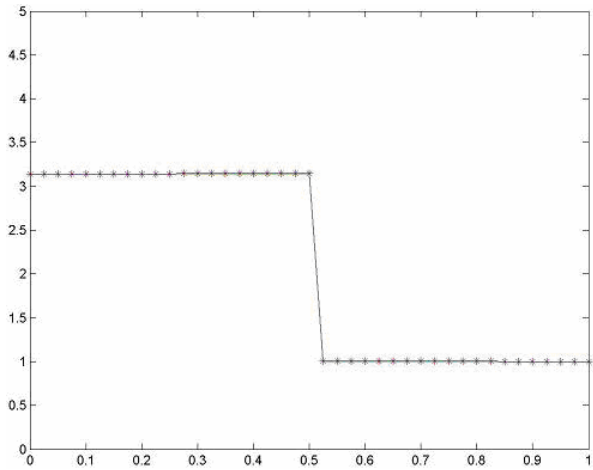

Fig. 1. Test $1-1(\delta=0)$

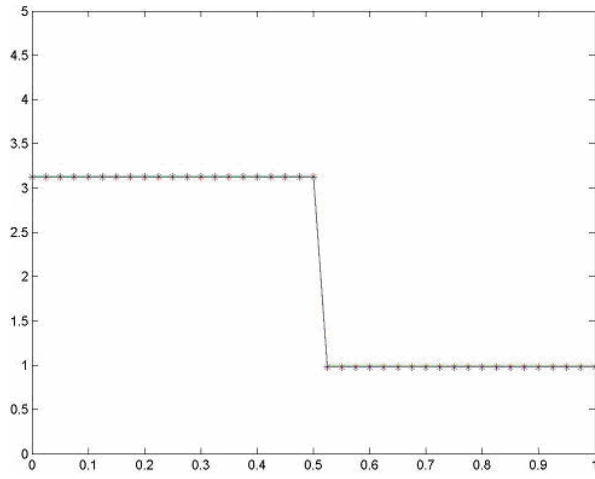

Fig. 2. Test $1-2(\delta=1 \%)$

Test 1-3: $\delta=5 \%, \beta=10^{-3}$. Run time is 197 seconds. The times for evaluating the discontinuous point are 26. The times for evaluating $q$ are 1042700. The noncontinuous point is $0.500000, \frac{\sqrt{\sum_{i=1}^{n-1}\left(u^{(b e s t)}\left(x_{i}\right)-\widetilde{u}\left(x_{i}\right)\right)^{2}}}{\sqrt{\sum_{i=1}^{n-1}\left(u^{(b e s t)}\left(x_{i}\right)\right)^{2}}}=0.027078$.

Test 1-4: $\delta=10 \%, \beta=10^{-3}$. Run time is 192 seconds. The times for evaluating the discontinuous point are 26. The times for evaluating $q$ are 1042700. The discontinuous point is $0.500000, \frac{\sqrt{\sum_{i=1}^{n-1}\left(u^{(\text {best }}\left(x_{i}\right)-\tilde{u}\left(x_{i}\right)\right)^{2}}}{\sqrt{\sum_{i=1}^{n-1}\left(u^{(b e s t)}\left(x_{i}\right)\right)^{2}}}=0.045741$. 


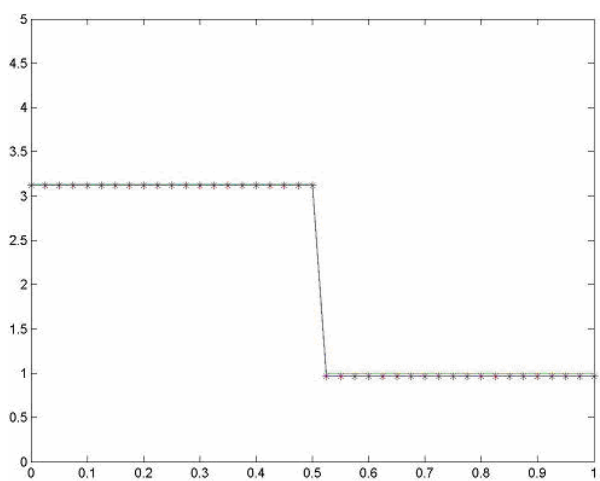

Fig. 3. Test $1-3(\delta=5 \%)$

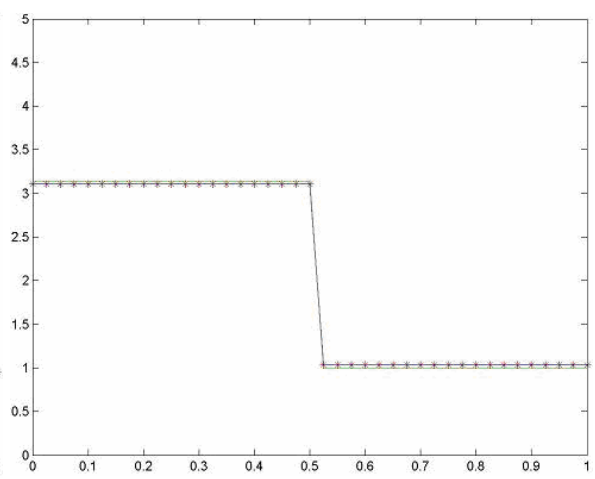

Fig. 4. Test $1-4(\delta=10 \%)$

\section{Example 2:}

$$
\left\{\begin{array}{l}
\frac{d}{d x}\left(q(x) \frac{d}{d x} u(x)\right)=f(x), x \in(0,1) \\
u(0)=0, u(1)=0
\end{array}\right.
$$

Where $q(x)=\left\{\begin{array}{l}\sin (\pi x)+1,0 \leq x \leq 0.5 \\ 4 x^{2}, 0.5<x \leq 1\end{array}, u(x)=\left\{\begin{array}{l}\sin (\pi x)+2,0 \leq x \leq 0.5 \\ 2 \sin (\pi x)+1,0.5<x \leq 1\end{array}\right.\right.$

$$
\tilde{u}_{i}=\left(1+\delta_{i}\right) u\left(x_{i}\right) \text {, where } \delta_{i}(i=0,1, \ldots n-1) \text { is a random number in }(-\delta, \delta)
$$

Test 2-1: $\delta=0, \beta=10^{-7}$. Run time is 116 seconds. The times for evaluating the discontinuous point are 12. The times for evaluating $q$ are 481300 . The discontinuous point is $0.500000, \frac{\sqrt{\sum_{i=1}^{n-1}\left(u^{(b e s t)}\left(x_{i}\right)-\tilde{u}\left(x_{i}\right)\right)^{2}}}{\sqrt{\sum_{i=1}^{n-1}\left(u^{(b e s t)}\left(x_{i}\right)\right)^{2}}}=0.000171$.

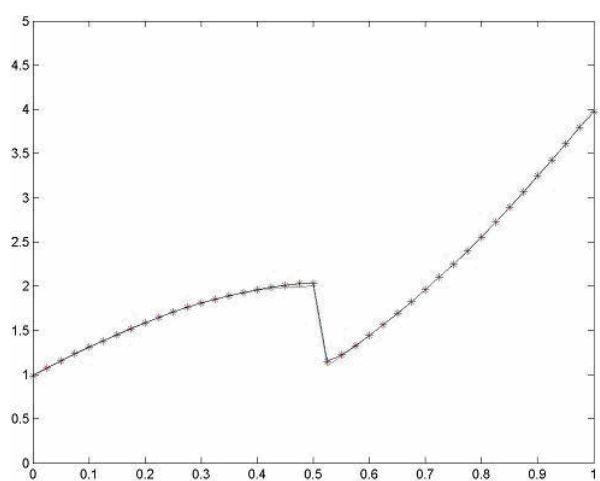

Fig. 5. Test 2-1( $\delta=0)$

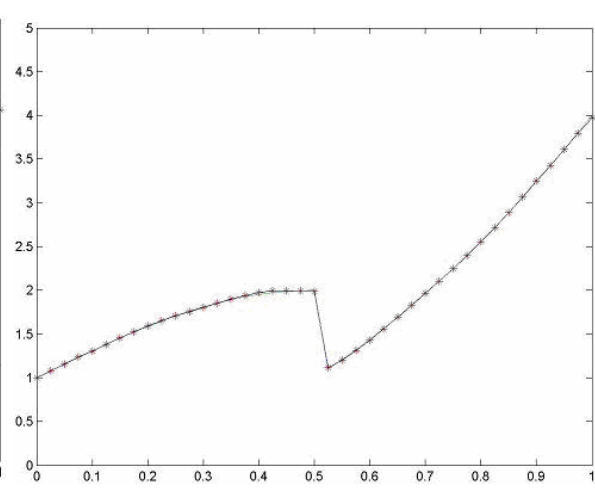

Fig. 6. Test 2-2( $\delta=1 \%)$ 
Test 2-2: $\delta=1 \%, \beta=10^{-7}$. Run time is 145 seconds. The times to evaluate the discontinuous point are 12 . The times to evaluate $q$ are 481300 . The non-continuous point is $0.500000, \frac{\sqrt{\sum_{i=1}^{n-1}\left(u^{(\text {best })}\left(x_{i}\right)-\tilde{u}\left(x_{i}\right)\right)^{2}}}{\sqrt{\sum_{i=1}^{n-1}\left(u^{(b e s t)}\left(x_{i}\right)\right)^{2}}}=0.00145^{\circ}$

Test 2-3: $\delta=5 \%, \beta=10^{-7}$. Run time is 258 seconds. The times for evaluating the discontinuous point are 26. The times for evaluating $q$ are 1042700. The noncontinuous point is $0.525000, \frac{\sqrt{\sum_{i=1}^{n-1}\left(u^{(\text {best })}\left(x_{i}\right)-\tilde{u}\left(x_{i}\right)\right)^{2}}}{\sqrt{\sum_{i=1}^{n-1}\left(u^{(b e s t)}\left(x_{i}\right)\right)^{2}}}=0.006168$.

Test 2-4: $\delta=10 \%, \beta=10^{-7}$. Run time is 621 seconds. The times for evaluating the discontinuous point are 38. The times for evaluating $q$ are 2343200. The noncontinuous is $0.475000, \frac{\sqrt{\sum_{i=1}^{n-1}\left(u^{(b e s t)}\left(x_{i}\right)-\tilde{u}\left(x_{i}\right)\right)^{2}}}{\left.\sqrt{\sum^{n-1}\left(u^{(b)}\right)}\left(x_{i}\right)\right)^{2}}=0.009756$.

$$
\sqrt{\sum_{i=1}^{n-1}\left(u^{(b e s t)}\left(x_{i}\right)\right)^{2}}
$$

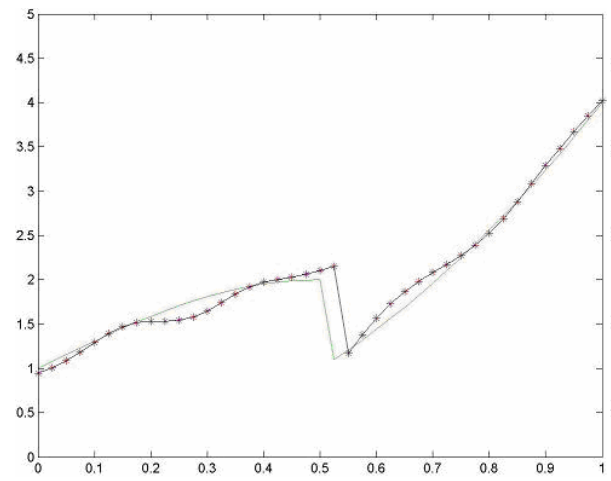

Fig. 7. Test 2-3( $\delta=5 \%)$

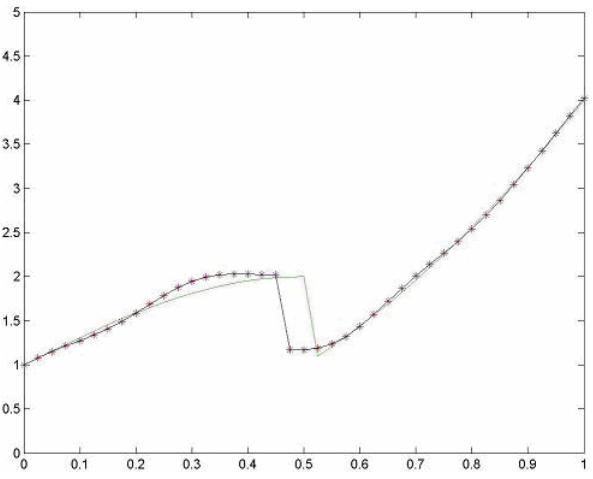

Fig. 8. Test 2-4( $\delta=10 \%)$

From the above described numerical experiments It can be seen that the parameter function found by our algorithm is very approximate to the original parameter. All numerical experiments have been conducted on PC

\section{Conclusions}

The results of our experiments suggest that the algorithm presented in this paper has a good appearance for inverse problems in which the coefficient $q(x)$ has discontinuous point. And what's more, the numerical experiments demonstrate that our algorithm carries the feature of prefect stability and adaptability and that it is not very sensitive to the noise, which tends to be the most important factors to parameter identification in the inverse problems. 
In is a new challenge to identify the discontinuous parameter by evolutionary algorithm. Only a simple case has been discussed in this paper, so in the further research, we will investigate some other kinds of inverse problems and model the discontinuous parameters by GP as well.

Acknowledgments. This research work was supported by Natural Science Foundation of Hubei Province (No. 2005ABA239)

\section{References}

1. Guo, B., and Zou, J. An augmented Lagrangian method for parameter identifications in parabolic systems. Journal of Mathematical Analaysis and Applications, 263 (2001) 49-68

2. Ito, K., and Kunisch, K. The augmented Lagrangian method for parameter estimation in elliptic system. SIAM J. Control Optim., 28(1990) 113-136

3. Keung, Y. L., and Zou, J. Numerical identifications of parameters in parabolic systems. Inverse Problems, 14 (1998) 83-100

4. Keung, Y. L., and Zou, J. An efficient linear solver for nonlinear parameter identification problems. SIAM J. Sci. Comput., 22, 5 (2000) 1511-1526

5. Xie, J., and Zou, J. Numerical reconstruction of heat fluxes. SIAM J. Numer. Anal. 43 (2005) 1504-1535

6. Burczynski, T., Beluch, W., Dlugosz, A., Orantek, P., and Nowakowski, M. Evolutionary methods in inverse problems of engineering mechanics. ISIP 2000 International Symposium on Inverse Problems in Engineering Mechanics (2000)

7. Collet, P., Lution, E., Raynal, F., and Schoenauer, M. Polar IFS+Parisian genetic programming=efficient IFS inverse problems. Genetic Programming and Evolvable Machines, 1 (2000) 339-361

8. Wu, Z., Tang, Z., Zou, J., Kang, L., and Li, M. Evolutionary algorithm for solving parameter identification problems in elliptic systems. In Proceedings of 2004 Congress on Evolutionary Computation, USA (2004) 803-808

9. Wu, Z., Tang, Z., Zou, J., Kang, L., and Li, M. An evolutionary algorithm for parameters identification in parabolic systems. In Proceedings of 2004 Genetic and Evolutionary Computation Conference, USA (2004) 1336-1337

10. Wu, Z., Kang L., Zou J., Tang Z., and Li, M. An evolutionary algorithm for identifying parameters in parabolic systems. In Progress in Intelligence Computation \& Applications, China, Wuhan (2005) 92-96

11. Cao, H., Kang, L. and Chen, Y. Evolutionary modeling of system of ordinary differential equations with genetic programming. Genetic Programming and Evolvable Machines, 1, 4(2000) 309-337

12. Koza, J. GeneticPprogramming: On the Programming of Computers by Means of Natural Selection. Cambridge, MA: MIT Press (1992)

13. Xiong, S., and Li Y. An evolutionary modeling approach of partial differential equations. Wuhan University Journal of Natural Sciences, 10, 5 (1999) 767-770

14. Xiong, S., and Lu X. A genetic programming approach to partial differential equation inverse problems. Journal of Wuhan University of Technology (Information \& Management Engineering), 25, 3 (2003) 11-15

15. Chen, Z., and Zou, J. An augmented Lagrangian method for identifying discontinuous parameters in elliptic problems. SIAM J. Control Optim., 37, 3(1999) 892-910 